\title{
RECOMBINATION NEARBY $N-n$ GaSb/GaInAsSb STAGGERED LINEUP HETEROJUNCTION
}

\author{
A.A. Popov, A.N. Imenkov, N.M. Kolchanova, Yu.P. Yakovlev \\ A.F. Ioffe Physico-Technical Institute, Russian Academy of Science \\ 26 Polytekhnicheskaya, 194021 St. Petersburg, Russia

\section{AND T.T. PIOTROWSKI} \\ Institute of Electron Technology, Al. Lotników 32/46, 02-668 Warszawa, Poland
}

The recombination nearby large band-offset staggered lineup $N$-GaSb/ $n$-GaInAsSb heterojunction was investigated by means of the electroluminescence and carrier lifetime measurements. It was demonstrated that the nature of recombination, tuning rate as well as relation between radiative and non-radiative recombination strongly depend on the $N-n$ band-offset and that its increase improves the carrier localization on the $N-n$ interface.

PACS numbers: $72.20 . \mathrm{Nz}$

The interactions in a staggered lineup (type-II) heterojunction are of interest since they exhibit unique optical and electronic characteristics [1]. These heterojunctions have been achieved within AlGaAs/GaAs superlattices [2] as well as within GaSb/GaInAsSb interfaces [3]. The potential wells for electrons and holes on both sides of interface are the consequence of the shape of the GaInAsSb conduction- and valence-band edges which are shifted downward to GaSb. Therefore there are possible direct transitions not only in the volume of the material but also along and across the $N$-GaSb/n-GaInAsSb interface. These could be radiative [3] as well as non-radiative [4] transitions. Heterostructures composed of GaInAsSb active layers lattice-matched to $\mathrm{GaSb}$ [5] meet the basic requirements for efficient LEDs and diode lasers working at room temperature in wavelength range from 1.7 to $2.4 \mu \mathrm{m}$. Therefore studying the recombination processes in the vicinity of the staggered lineup type-II GaSb/GaInAsSb heterojunction which in turn can offer unique possibilities for device applications is a very important goal.

The purpose of this paper is to investigate the nature of recombination from the $N$-GaSb $/ n$-GaInAsSb/P-GaAlAsSb heterostructures with varied band-offset at $N-n$ staggered lineup interface.

Figure 1 shows the energy band diagram of the heterostructures with different band-gap energy of a buffer layer. The structures were fabricated by liquid-phase epitaxy technology described in [4]. 
(1)

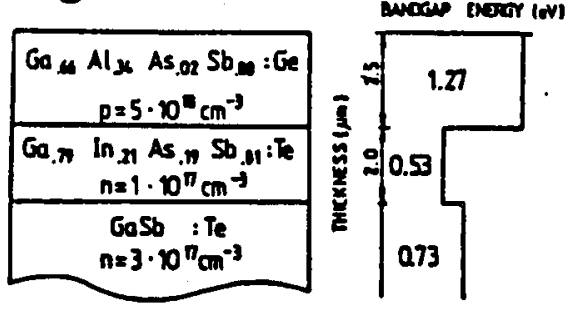

(11)
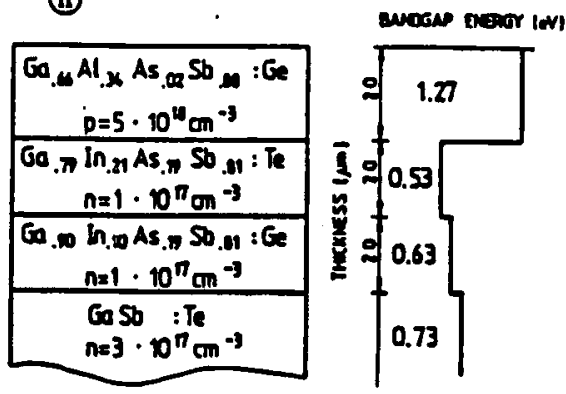

畐
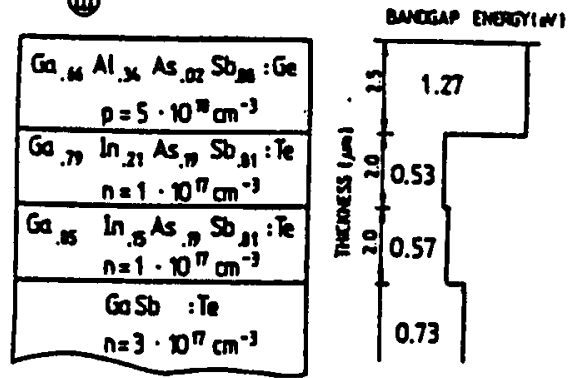

Fig. 1. Schematic diagram of $N-G a S b / n-G a I n A s S b / P-G a A l A s S b$ heterostructures and their respective band-gap profiles.

The electroluminescence (EL) spectra, optical output, external quantum yield and relative lifetime of carriers were investigated in the temperature range of $77-300 \mathrm{~K}$. The measurements were performed under DC (up to $150 \mathrm{~mA}$ ) and 150 ns pulsed (over $0.15-3 \mathrm{~A}$ ) current.

The EL spectra exhibited at least two peaks: $A$ and $B$. Both peaks were shifted towards short wavelengths and depend on the $N-n$ offset magnitude (Fig. 2a). The position of emission peak for band $A$ is close to the band gap of active layer, the parabolic shape of spectra is a consequence of the band-to-band recombination. The shift rate is close to the change of the quasi-Fermi levels position. For the large offset heterostructure I the long-wavelength band $A^{\prime}$ occurred under current smaller than $100 \mathrm{~mA}$ at $300 \mathrm{~K}$ and for current smaller than $30 \mathrm{~mA}$ at $77 \mathrm{~K}$. The nature of the recombination for band $A^{\prime}$ is different [6] than for band $A$ because of the presence of the tail on the long-wavelength side of the spectra and TE polarization of emission.

The carrier lifetime value was as low as $3 \mathrm{~ns}$ for thin layer (Fig. 2b), i.e. about three times smaller than for band-to-band recombination. Introducing the buffer layer into the structures which corresponds to the offset decrease, one causes the increase in the carrier lifetime up to the value resulting from the band-to-band transitions (Fig. 2c).

Under high current the rapid-tuning short-wavelength band $B$ appeared. The peak energy of band $B$ was tuned to the buffer band-gap energy and exceeded 

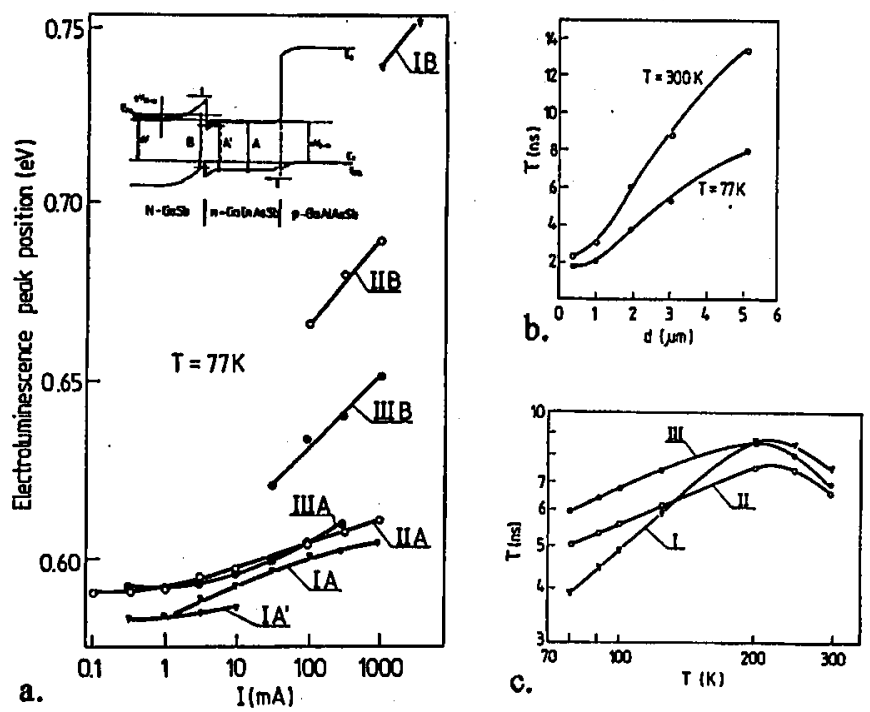

Fig. 2. (a) Current dependence of the energy position of the EL peak $A$ and $B$ at $77 \mathrm{~K}$ for heterostructures I, II and III. The inset shows the energy-band diagram of the forward-biased $N$-GaSb $/ n-\mathrm{GaInAsSb} / P-\mathrm{GaAlAsSb}$ heterostructure and possible radiative transitions: band-to-band recombination in the active layer $(A)$ and interface recombinations along $\left(A^{\prime}\right)$ and across $(B)$ heteroboundary. (b) Carrier lifetimes at $77 \mathrm{~K}$ and $300 \mathrm{~K}$ as a function of the distance between $p-n$ junction and $N-n$ interface as measured for heterostructure I. (c) Carrier lifetimes for the heterostructures I, II and III as a function of heat sink temperature.

the active band-gap energy by $30-150 \mathrm{meV}$. The tuning rate was faster and the threshold of appearance was smaller for the heterostructures with large $N-n$ offset and at low temperatures. The analysis indicates that band $B$ associated with recombination by tunneling across the $N-n$ interface results in tunable emission.

At $T=77 \mathrm{~K}$ the optical output $P$ increases with current $I$ as $P \sim I^{n}$ with $n$ decreasing from 4 to 1 as $I$ increases between 1 and $1000 \mathrm{~mA}$. At $T=300 \mathrm{~K}$ the value of $n$ decreases from 2 to 0.5 as the current increases from $3 \mathrm{~mA}$ to $1.5 \mathrm{~A}$. The highest optical output was obtained for heterostructure II and the smallest for heterostructure I. The external quantum yield is determined by competition between radiative and non-radiative Auger recombination via both bulk and interface transitions. At high temperatures when the offset is large, the quantum yield decreases strongly as a consequence of the interface Auger recombination [7]. Introduction of a buffer layer which diminishes the $N-n$ offset magnitude and also diminishes potential wells, leads to decrease in Auger interface recombination. This is the reason why the maximal yield occurred for heterostructure II and minimal for heterostructure I. Above $T=110 \mathrm{~K}$ the Auger recombination causes the increase in quantum yield as $n^{-3 / 2}$.

In conclusion, it has been demonstrated that the recombination nearby the $N-n \mathrm{GaSb} / \mathrm{GaInAsSb}$ staggered lineup heterojunction dominates over the band- 
-to-band recombination. It appeared that the increase in the $N-n$ band-offset in a wide current range leads to the improvement of carrier localization in the vicinity of the interface and therefore to the increase in interface transitions. Under small drive current the recombination along $N-n$ interface occurred. Under higher current the recombination by tunneling across $N-n$ interface dominates, resulting in rapid current-tunable position of emission peak. It has been demonstrated that the $N-n$ offset leads to amplification of both radiative and Auger recombinations at the interface.

\section{References}

[1] B.A. Wilson, IEEE J. Quantum Electron. QE-24, 1763 (1988).

[2] G. Danan, F.R. Ladan, F. Mollot, R. Planel, J. Phys. (Paris) C 5, 499 (1987).

[3] A.N. Baranov, A.N. Imenkov, M.P. Mikhailova, A.A. Rogachev, A.N. Titkov, Yu.P. Yakovlev, Superlattices Microstruct. 8, 375 (1990).

[4] N.M. Kolchanova, A.A. Popov, A.B. Bogoslovskaya, G.A. Sukach, Sov. Tech. Phys. Lett. 19, 61 (1993).

[5] A.A. Andaspaeva, A.N. Baranov, A.A. Guseinov, A.N. Imenkov, N.M. Kolchanova, E.A. Sidorenkova, Yu.P. Yakovlev, Sov. Tech. Phys. Lett. 14, 377 (1988).

[6] A.A. Monahov, A.A. Rogachev, Solid State Phys. 32, 778 (1990).

[7] G.G. Zegrya, V.A. Kharchenko, Sov. Phys.-JETP 100, 432 (1992). 